\title{
Proposal of Noninvasive Liver Function Measurement Method via Saliva
}

\author{
Masaki Yamaguchi* ${ }^{*}$ Member \\ Yuji Kawabata* Non-member \\ Toyomasa Hatakeyama* Member \\ Yoshiro Kashii** Non-member
}

\begin{abstract}
The authors studied the correlation between serum alanine aminotransferase (ALT) activity and salivary ALT activity using ten healthy young adults and ten liver disease patients. Firstly, in order to establish the experimental conditions, we investigated the influence of occult blood and salivary secretion rate on the salivary ALT activity using healthy subjects. Then, simultaneous analysis of the serum and salivary ALT activities were conducted to investigate the correlation using the twenty subjects. As the results, although salivary ALT activity was as low as one third of serum ALT activity, the presence of salivary ALT activity was confirmed in healthy young adults whose saliva was not contaminated with serum. The salivary ALT activity of liver disease patients showed higher values than that of healthy young adults. In other word, if a threshold of salivary ALT activity was established, healthy young adults could be distinguished from liver disease patients.
\end{abstract}

Keywords : noninvasive, liver function, saliva, alanine aminotransferase, screening

\section{Introduction}

It is difficult to diagnose three major hepatic diseases, hepatitis, liver cirrhosis and liver cancer, in their early stages since there are scarcely any noticeable specific symptoms. Hence, the liver is called " a silent organ". Therefore, screenings for hepatic diseases have been conducted using serum enzymes such as alanine aminotransferase (ALT) and asparate aminotransferase (AST) ${ }^{(1)}$. A procedure requiring blood samples, however, risks causing virus infections, so it is not desirable for a liver function measurement test. In addition, without a noninvasive liver function measurement method, there also is a risk of not finding liver dysfunction caused by antibiotic dose before the condition becomes serious. If an appropriate liver function measurement method that patients could easily operate at home were to be developed, it would help to find the symptoms before they become serious, even allowing a longer period between medical doctor's tests.

The authors have been studying the development of an easy to operate noninvasive liver function measurement method focusing on the serum enzymes that are secreted in oral fluid such as saliva, specifically, ALT activities ${ }^{(2)}$. ALT is an enzyme presents in the liver cells, as are AST and $\gamma$-GTP. The concentration of ALT in blood increases when liver cell necrotize or degenerate. Because of this feature, it is a widely used index for the estimation of the liver malfunction level. AST in saliva is activated with periodontal disease, while ALT is mostly present in the liver, and is specific to liver function compared with other enzymes ${ }^{(3) \sim(5)}$.

In order to determine the validity of the liver function

\footnotetext{
* Faculty of Engineering, Toyama University

3190 Gofuku, Toyama 930-8555

** Toyama Saiseikai Hospital

33-1 Kusunoki, Toyama 931-8442
}

measurement method, we studied the correlation between serum ALT activity and salivary ALT activity using ten healthy young adults and ten liver disease patients; twenty subjects in total. Firstly, in order to establish the experimental conditions, we investigated the influence of occult blood and salivary secretion rate on the salivary ALT activity using healthy subjects. Then, simultaneous analysis of the serum and salivary ALT activities were conducted to investigate the correlation using the twenty subjects.

\section{Materials and Methods}

\subsection{Influence of Occult Blood on Saliva ALT Activity}

The subjects were ten healthy young adults who had no liver disease (seven male and three female, aged between 21 and 25 years old, Table 1). The body mass indexes (BMI) were in the range from 17.2 to $22.3 \mathrm{~kg} / \mathrm{m}^{2}$. The serum ALT activities were in

Table 1. Background of healthy young adults.

\begin{tabular}{c|cccc}
\hline \hline Subjects & Sex & Age & $\begin{array}{c}\text { BMI } \\
\left(\mathrm{kg} / \mathrm{m}^{2}\right)\end{array}$ & $\begin{array}{c}\text { Serum ALT } \\
\text { activity (IU/L) }\end{array}$ \\
\hline $\mathrm{a}$ & $\mathrm{M}$ & 22 & 21.0 & 21 \\
$\mathrm{~b}$ & $\mathrm{M}$ & 24 & 20.6 & 18 \\
$\mathrm{c}$ & $\mathrm{M}$ & 23 & 20.0 & 18 \\
$\mathrm{~d}$ & $\mathrm{~F}$ & 21 & 19.7 & 16 \\
$\mathrm{e}$ & $\mathrm{M}$ & 22 & 22.3 & 15 \\
$\mathrm{f}$ & $\mathrm{M}$ & 25 & 20.5 & 13 \\
$\mathrm{~g}$ & $\mathrm{~F}$ & 21 & 21.4 & 12 \\
$\mathrm{~h}$ & $\mathrm{M}$ & 22 & 18.7 & 11 \\
$\mathrm{i}$ & $\mathrm{M}$ & 21 & 21.2 & 10 \\
j & $\mathrm{F}$ & 22 & 17.2 & 8 \\
\hline Mean & - & $22.3 \pm 1.3$ & $20.3 \pm 1.5$ & $14.2 \pm 4.1$ \\
\pm SD & \multicolumn{4}{l}{} \\
M: male, F:female &
\end{tabular}




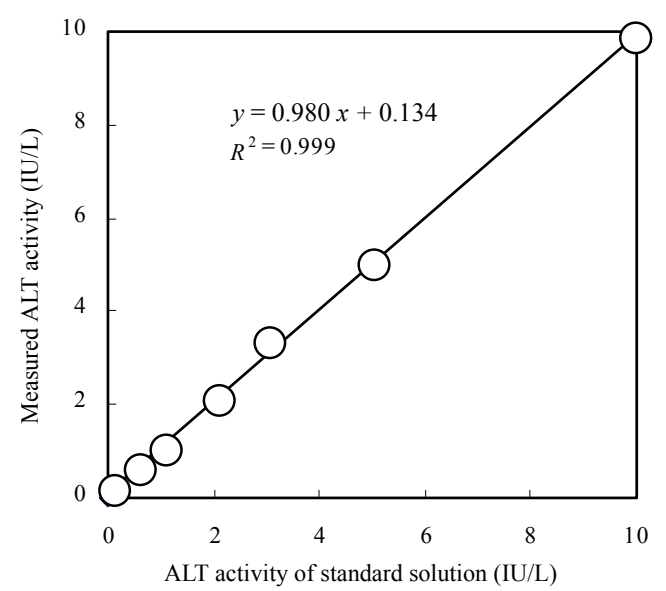

Fig. 1. Calibration curve of the ALT activity used for the measurement of salivary ALT activity.

the range from 8 to $21 \mathrm{IU} / \mathrm{L}$. These values were all in the normal range. The experiment was carried out during the morning, over 2 hours after breakfast. The aim of the experiment was explained to the subjects and consent was obtained after confirmation that they fully understood the experiment.

Whole saliva was collected in nonstimulation conditions, then both salivary hemoglobin concentration (salivary $\mathrm{Hb}$ ) and salivary ALT activity were measured in order to confirm the existence of disease around the teeth. Prior to collection of the saliva, the subjects brushed their teeth clean and completely rinsed the mouth to remove any residues. All the water that remained in the mouth was wiped out with a dental cotton ( $8 \mathrm{~mm}$ diameter, $25 \mathrm{~mm}$ length). Next, the subjects were made to take a sitting position in a silent room. Then, a cotton roll was set under the tongue, and left in place for three minutes in order to collect the whole saliva. Immediately after, the cotton roll was condensed using a syringe and about $8 \mathrm{~mL}$ of whole saliva was collected in a glass bottle.

The salivary $\mathrm{Hb}$ was measured using $20 \mu \mathrm{L}$ of whole saliva, sodium lauryl sulfate hemoglobin method reagent (Wako Pure Chemical Industries, Ltd., Hemoglobin $\mathrm{B})^{(6)}$, and a spectrophotometer (Hitachi Ltd, U-3010). The salivary ALT activity was measured using $30 \mu \mathrm{L}$ of whole saliva, UV-method reagent for serum use (Wako Pure Chemical Industries, Ltd., L-Type GPT $\cdot \mathrm{J} 2)^{(7)}$ and a clinical automatic analyzer ((Nipro Co., MIRACLE ACE 919). To improve the accuracy, the measurement of salivary ALT activity was repeated three times, and the mean value was obtained. One unit activity per volume (IU/L) will convert $1.0 \mu \mathrm{mol}$ of $\alpha$-ketoglutarate to L-glutamate per min at $\mathrm{pH} 7.6$ at $25^{\circ} \mathrm{C}$ in the presence of L-alanine. $R^{2}$ and $\mathrm{CV}$ of the calibration curve were 0.999 and 8.0, respectively (Fig.1).

2.2 Salivary Secretion Rate The subjects were the same healthy young adults that took part in the occult blood test. The occult blood test and the measurement of salivary secretion rate were performed in the same day. Prior to collection of saliva, the subjects brushed their teeth clean and completely rinsed the mouth to remove any residues. All the water that remained in the mouth was wiped out with dental cotton. Next, the subjects were made to take a sitting position in a silent room. Then, the cotton roll was set under the tongue, and left in place for three minutes in order to collect the whole saliva. Immediately after, the cotton
Table 2. Background of liver disease patients.

\begin{tabular}{c|cccc}
\hline \hline Subjects & Sex & Age & $\begin{array}{c}\text { BMI } \\
\left(\mathrm{kg} / \mathrm{m}^{2}\right)\end{array}$ & $\begin{array}{c}\text { Serum ALT } \\
\text { activity (IU/L) }\end{array}$ \\
\hline A & M & 75 & 22.7 & 82 \\
B & M & 62 & 22.1 & 68 \\
C & M & 54 & 18.2 & 68 \\
D & M & 31 & 21.6 & 42 \\
E & F & 56 & 21.6 & 42 \\
F & M & 61 & 18.4 & 41 \\
G & F & 47 & 24.4 & 41 \\
H & M & 59 & 24.2 & 35 \\
I & M & 39 & 20.7 & 27 \\
J & F & 66 & 24.5 & 26 \\
\hline Mean & - & $55.0 \pm 13.0$ & $21.8 \pm 2.3$ & $47.2 \pm 18.9$ \\
\pm SD & \multicolumn{5}{|}{} \\
\hline \hline \\
M: male, F:female
\end{tabular}

roll was condensed using a syringe and a few $\mathrm{mL}$ of whole saliva was collected in a glass bottle. This operation was repeated five times, so the salivary secretion rate was measured throughout fifteen minutes in total. The mass of the whole saliva was measured using an electronic analytical balance (A\&D Co. Ltd, HM-202, $0.01 \mathrm{mg}$ of optimum sensitivity) in order to measure the collection quantity in each sampling time. Then, the volume was estimated from the mass setting the specific gravity to be 1 . That is to say, $0.1 \mathrm{mg}$ correspond to $0.1 \mu \mathrm{L}$. Finally, the salivary ALT activity was also measured using the whole saliva collected throughout fifteen minutes.

2.3 Salivary ALT Activity The subjects were ten liver disease ambulatory patients who had no oral disease (seven male and three female, aged between 31 and 75 years old, Table 2). Their BMIs were in the range from 18.2 to $24.5 \mathrm{~kg} / \mathrm{m}^{2}$. Three patients who had improved liver function were included in order to verify the validity of the liver function measurement method. The results showed that the serum ALT activities were in the range from 26 to $82 \mathrm{IU} / \mathrm{L}$. The experiment was carried out during the morning, over 2 hours after breakfast. The aim of the experiment was explained to the subjects and consent was obtained after confirmation that they fully understood the experiment.

The collection protocol of the whole saliva and the measurement method of salivary ALT activity were the same as for the healthy young adults. The serum ALT activity was also measured by collecting the blood simultaneously (Table2).

\section{Results and Discussion}

\subsection{Influence of Occult Blood on Saliva ALT Activity}

The values of salivary $\mathrm{Hb}$ for healthy young adults ranged from 0.04 to $0.81 \mathrm{mg} / \mathrm{dL}$. They were four digits lower than that of the standard value for serum $\mathrm{Hb}$ (male: from 13.1 to $17.0 \mathrm{~g} / \mathrm{dL}$ and female: from 11.5 to $14.5 \mathrm{~g} / \mathrm{dL})^{(8)}$ (Fig. 2). All of the measured salivary $\mathrm{Hb}$ values were lower than that of the standard value of $1.0 \mathrm{mg} / \mathrm{dL}$ for suspected periodontal disease ${ }^{(9)}$. These results showed that the subjects were also healthy with regard to oral diseases.

The values of salivary ALT activity were in the range from 0.8 to $9.5 \mathrm{IU} / \mathrm{L}$ while the serum ALT activities were in the range from 8 to $21 \mathrm{IU} / \mathrm{L}$ (mean: $14.2 \mathrm{IU} / \mathrm{L}$, Table 1 ). Although both values were in almost the same density range, salivary ALT activity 


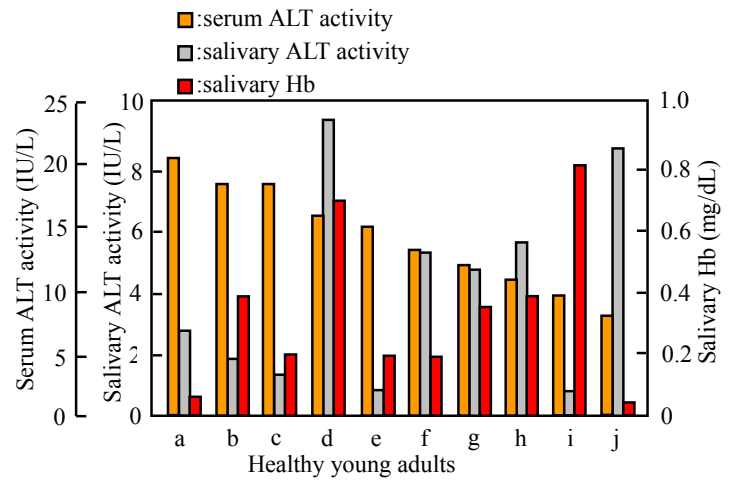

Fig. 2. ALT activities and salivary $\mathrm{Hb}$ of the healthy young adults.

showed lower value and its mean value was one third of the serum ALT. In other words, salivary ALT activity was confirmed for the healthy young adults whose serum was not contaminated.

3.2 Salivary Secretion Rate The mean value of the salivary secretion rate for 15 minutes was in the range from 1.19 to $4.76 \mathrm{~mL} / \mathrm{min}$ for each healthy young adult (Fig. 3 (a)). Except subjects $\mathrm{c}$ and $\mathrm{f}$, the mean value and $\mathrm{CV}$ were $1.9 \mathrm{~mL} / \mathrm{min}$ and $21.1 \%$ and significant difference among the healthy young adults was not observed.

The mean value for salivary ALT activity (IU/L) for 15 minutes was in the range from 0.7 to $9.7 \mathrm{IU} / \mathrm{L}$ and tenfold difference between the minimum and the maximum values was observed (Fig 3 (b)). The correlation between salivary secretion rate and salivary ALT activity was 0.03 . It indicated that there was no significant correlation between them.

Then, to consider the influence of salivary secretion rate on the concentration of chemical substances presented in saliva, salivary secretion rate $(\mathrm{mL} / \mathrm{min})$ and salivary ALT activity (IU/L) values were added up and the value for the salivary ALT activity (IU/min) per minute was calculated in order to offset the influence (Fig 3 (c)). The correlation between salivary ALT activity and salivary ALT activity per minute was 0.03 . In this case no meaningful correlation was observed. The influence of salivary secretion rate on the salivary ALT activity cannot be completely neglected, however, a significant influence on whole saliva collection under the quiet circumstances was not observed.

During in vivo evaluation at a hospital, it is difficult to regularly measure salivary secretion rates for the subjects with liver disease due to the need for consideration of the patients' condition. Therefore, the salivary secretion rate for the liver disease subjects was not conducted.

3.3 Salivary ALT Activity For both healthy young adults and liver disease patients, serum ALT activity was in the range from 8 to $82 \mathrm{IU} / \mathrm{L}$ (mean: $30.7 \mathrm{IU} / \mathrm{L}$ ) while salivary ALT activity was from 0.8 to $31.9 \mathrm{IU} / \mathrm{L}$ (mean: $9.5 \mathrm{IU} / \mathrm{L}$ ). Both values were almost in the same concentration range, but salivary ALT activity was lower than serum ALT activity and the comparison of their mean values exhibited a difference of one third (Fig. 4).

The mean values of ALT activity of serum and saliva of the healthy young adults was $14.2 \pm 4.1$ and $4.4 \pm 2.9 \mathrm{IU} / \mathrm{L}$, respectively. The mean values of ALT activity of serum and saliva of the liver disease patients was $47.2 \pm 18.9$ and $14.6 \pm$ $9.4 \mathrm{IU} / \mathrm{L}$, respectively. Except for one recovering liver disease patient (subject J), the liver disease patients showed higher values

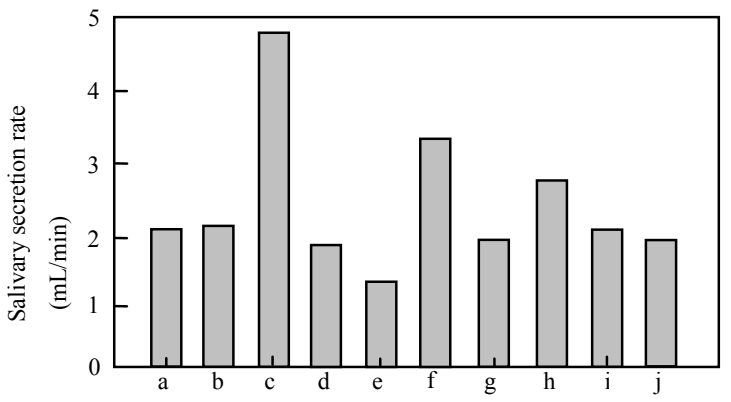

(a) Salivary secretion rate (mean value)

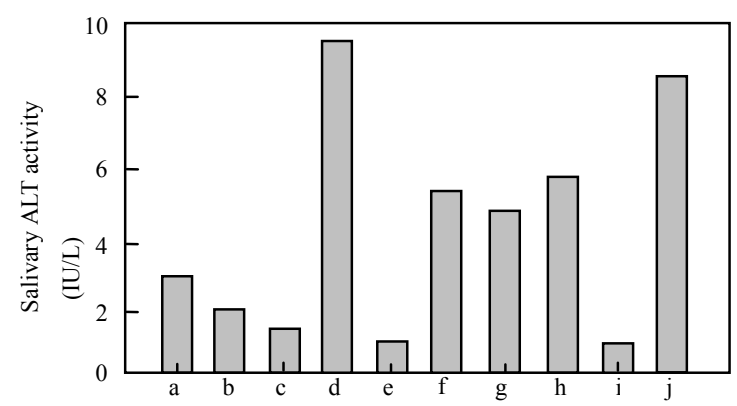

(b) Salivary ALT activity

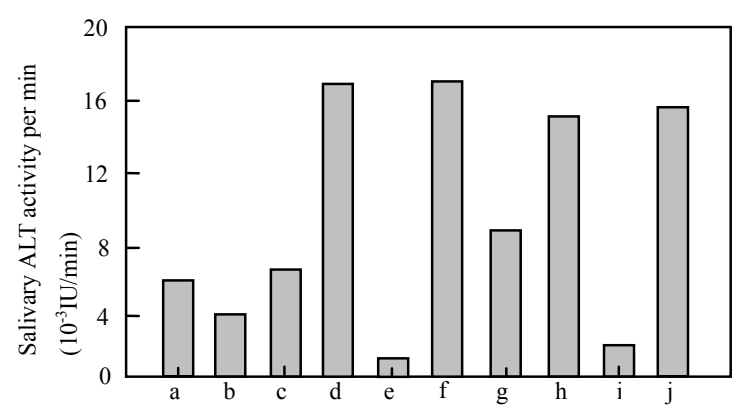

(c) Salivary ALT activity per min

Fig. 3. Measured results of salivary secretion rate and salivary ALT activity of healthy young adults.

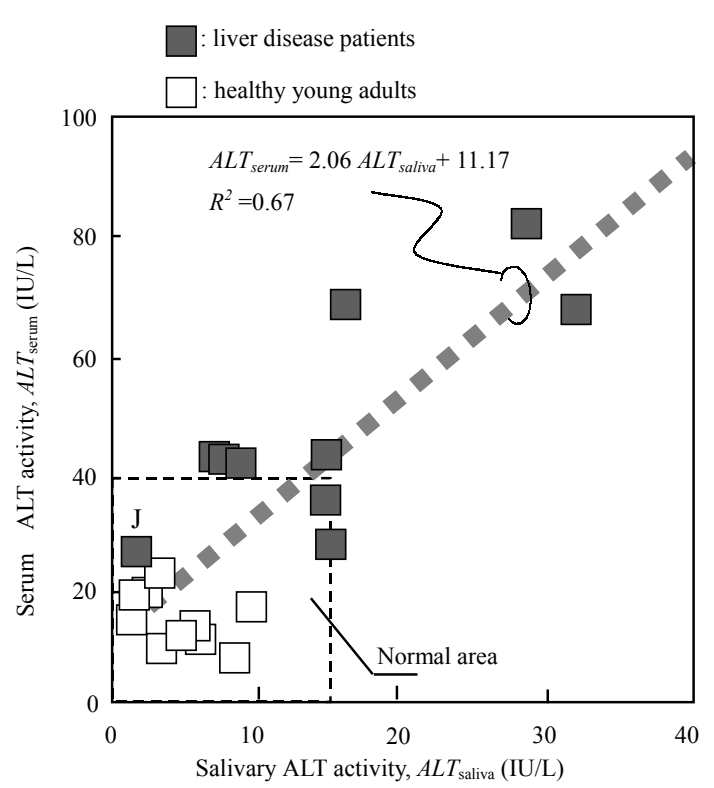

Fig. 4. Relationship of the ALT activities between serum and saliva (20 subjects). 
compared with the healthy young adults for not only serum but also salivary ALT activities.

As the normal value for serum ALT activity is lower than 40 $\mathrm{IU} / \mathrm{L}$, when the threshold for salivary ALT activity was $14 \mathrm{IU} / \mathrm{L}$, healthy young adults could be distinguished from liver disease patients. Further study with larger number of subjects and optimization of the threshold should be expected.

\section{Conclusion}

In order to develop a convenient noninvasive liver function measurement method, the correlation between serum ALT activity and salivary ALT activity was investigated using 10 healthy young adults and 10 liver disease patients. The experimental results revealed the following:

1. Although salivary ALT activity was as low as one third of serum ALT activity, the presence of salivary ALT activity was confirmed in healthy young adults whose saliva was not contaminated with serum.

2. Significant influence of salivary secretion rate on the salivary ALT activity was not observed, when whole saliva was collected from the healthy young adults under quiet circumstances without any stimulation.

3. The salivary ALT activity of liver disease patients showed higher values than that of healthy young adults. In other word, if a threshold of salivary ALT activity was established, healthy young adults could be distinguished from liver disease patients.

Since several methods based on biosensors in order to measure the enzyme activity present in saliva have been developed ${ }^{(10)}$, as the next step, it will be important to develop a biosensor for salivary ALT activity which has the advantages of simplicity, any-time use and immediacy.

(Manuscript received Jan. 30, 2003, revised April 11, 2003)

References

(1) T. Murai, M. Mori, N. Sato, and K. Ito : "Introduction of Clinical Laboratory Medicine”, Nanzando Co. Ltd., Tokyo, p.45-46 (2000)

(2) M. Yamaguchi, Y. Kawabata, Y. Kashii, and T. Osaka : "Comparison between saliva and gingival crevicular fluid as an index for liver function", $2^{\text {nd }}$ Joint EMBS-BMES Conference, pp. 1767-1768 (2002)

(3) D.A. Chambers, J.M.Crawford, S. Mukherjee, and R.L. Cohen : "Asparate aminotransferase increases in crevicular fluid during experimental periodontitis in beagle dogs", J Periodontol., Vol.55, No.9, pp.526-530 (1984)

(4) P.B.Imrey, J.M.Crawford, R.L.Cohen, M.E.A.F.Alves, T.A.McSwiggin, and D.A.Chambers : "A cross-sectional analysis of aspartate aminotransferase in human gingival crevicular fluid", $J$ Periodont Res, Vol.26, pp.75-84 (1991)

(5) T. Nakamura, J. Kido, R. Kido, K. Ohishi, N. Yamauchi, M. Kataoka, and T. Nagata : "The association of calprotectin level in gingival crevicular fluid with gingival index and the activities of collagenase and aspartate aminotransferase in adult periodontitis patients", J Periodontal, Vol.71, No.3, pp.361-367 (2000)

(6) I. Oshiro, M. TsutsuI, M. Fujii, M. Ueyama, T. Takenaka, and J. Maeda : "New Manual and Automatical Method of Hemoglobin Determination by Using SLS", The Japanese Journal of Clinical Pathology, Vol.29, No.2, pp.203-209 (1981)

(7) Instruction sheet : L-type GPT $\cdot$ J2(JSCC Transferable Method),
REV\#3/0105IBDOOK, Wako Pure Chemical Industries, Ltd.(2001)

(8) S. Matsuo and Y. Maekawa : "Clinical Laboratory Dictionary", Medicus Shuppan, Osaka, p.282 (1998) (in Japanese)

(9) S. Sano : "Study on gingivitis screening using salivary occult blood test paper in mass examination", Journal of Dental Health, Vol.40, No.3, pp.305-318 (1990) (in Japanese)

(10) M. Yamaguchi, M. Kanemaru, T. Kanemori, and Y. Mizuno : "Flow-injection-type biosensor system for salivary amylase activity", Biosensors \& Bioelectronics, Vol.18, No5-6, pp.835-840 (2003)

Masaki Yamaguchi (Member) was born in Nagoya, Japan, on

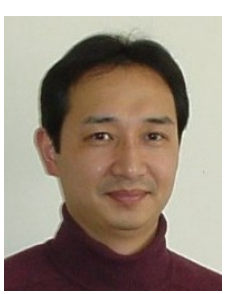
28th January, 1963. He received the B.S. and M.S. degrees in engineering from Shinshu University, Japan, in 1985 and 1987. He joined the Research Laboratory of Brother Industries, LTD., Japan, in 1987. He received the doctoral degree in engineering from Shinshu University in 1994 . He started an assistant professor at Tokyo University of Agriculture and Technology since 1995. He is currently working as an associate professor in faculty of engineering at Toyama University since 1999. His primary research interests focus on the development of noninvasive medical sensors and welfare apparatuses. In 2001 he received Nikkei BP Technology Awards in Medicine/ Biotechnology Section from Nikkei Business Publications, Inc. He studied as a visiting scientist at Linköping University in 2002. $\mathrm{He}$ is a member of the IEEE EMBS, the Japan Society of Medical Electronics and Biological Engineering (JME).

Yuji Kawabata (Non-member) was born in Ishikawa, Japan, on

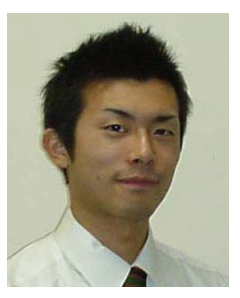
30th September, 1979. He received the B.S. degrees from Toyama University in 2002. $\mathrm{He}$ is a member of the IEEE and the Japan Society of Medical Electronics and Biological Engineering (JME).

Toyomasa Hatakeyama (Member) was born in Toyama, Japan,

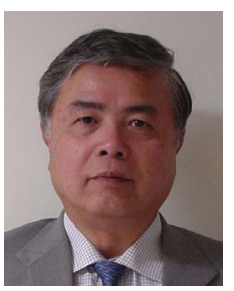
in 1941. He received the M.S. degree in science from Kanazawa University in 1966 and obtained his Doctor degree in Engineering from Tokyo Institute of Technology in 1981. $\mathrm{He}$ is a Professor in the Dept. Material Systems Engineering and Life Science of Toyama University. $\mathrm{He}$ is engaged in research on bioelectricity engineering, and biophysics using computer simulation.

Yoshiro Kashii (Non-member) was born in Toyama, Japan, on

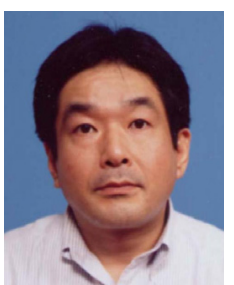
1st September, 1962. He graduated from Toyama Medical and Pharmaceutical University in 1989. He had studied as a postdoctoral fellow at University of Pittsburgh Cancer Institute from 1995 to $1997 . \mathrm{He}$ is working as a medical stuff in Toyama Saiseikai hospital. $\mathrm{He}$ is a member of the Japan Society of Hepatology. 